\title{
"I must be cruel only to be kind ». Le concept de dignité humaine chez Montaigne et chez Shakespeare
}

Jean-François Chappuit

\section{(2) OpenEdition}

\section{Journals}

Édition électronique

URL : http://journals.openedition.org/shakespeare/150

DOI : 10.4000/shakespeare.150

ISSN : 2271-6424

Éditeur

Société Française Shakespeare

Édition imprimée

Date de publication : 1 novembre 2004

Pagination : 53-78

ISBN : 2-9521475-0-7

Référence électronique

Jean-François Chappuit, « «I must be cruel only to be kind ». Le concept de dignité humaine chez Montaigne et chez Shakespeare ", Actes des congrès de la Société française Shakespeare [En ligne], $21 \mid$ 2004, mis en ligne le 27 janvier 2007, consulté le 10 décembre 2020. URL : http:// journals.openedition.org/shakespeare/150; DOI : https://doi.org/10.4000/shakespeare.150 


\section{Shakespeare et Montaigne vers un nouvel humanisme}

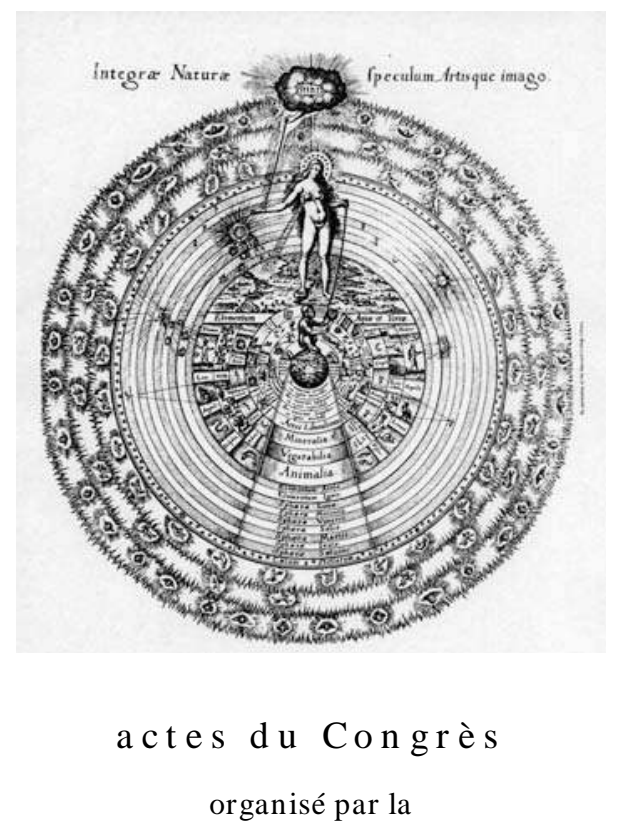

SOCIÉTÉ FR ANÇAISE SH AKESPEARE en collaboration avec la

S OC I É T É I N T E R N A T I O N A L E DES AM I D E M O N A I G N les 13,14 et 15 mars 2003

$$
\begin{gathered}
\text { textes réunis par } \\
\text { Pierre KAPITANIAK } \\
\text { sous la direction de } \\
\text { Jean-Marie MAGUIN }
\end{gathered}
$$




\section{COMITÉ SCIENTIFIQUE :}

Margaret Jones-Davis

Gisèle Venet

Jean-Marie Maguin

Yves Peyré

François Laroque

Pierre Kapitaniak

\section{COUVERTURE :}

Robert Fludd

Utriusque Cosmi Historia (1617-19)

planche 17

conception graphique et logo

Pierre Kapitaniak

\section{(C) 2003 Société Française Shakespeare}

Institut du Monde Anglophone

Université de Paris III - Sorbonne Nouvelle

http:// univ-montp3.fr/SFS/

5 rue de l'École de Médecine

75006 Paris

Diffusion :

AVL DIFFUSION

Parc Euromédecine

34198 MONTPELLIER CEDEX 5

ISBN 2-9521475-0-7

Tous droits de traduction, de reproduction et d'adaptation réservés pour tous les pays 


\title{
«I MUST BE CRUEL ONLY TO BE KIND » LE CONCEPT DE DIGNITÉ HUMAINE CHEZ Montaigne ET CHEZ SHAKeSPEARE
}

\author{
Jean-François CHAPPUIT
}

\begin{abstract}
L'humanisme, selon Olivier Boulnois, marque un point d'inflexion entre la pensée médiévale et la pensée moderne des rapports entre Dieu, l'homme et le monde. Pic de La Mirandole, Érasme, pour ne citer qu'eux, tirent de ce qui est alors appelé la philosophie chrétienne, la notion de dignité humaine intrinsèque à l'homme. Par le libre arbitre, don de Dieu, l'homme peut dépasser sa nature animale et recouvrer son essence divine dans l'épopteia, ou, pour reprendre les termes de Pétrarque, passer de homo à vir. La dignité humaine s'affirme par le truchement de la force (virtù) et de la vertu nécessaires à l'homme dans sa lutte permanente contre les forces du Mal qui assaillent sa station. À la même époque, les Réformateurs et notamment Calvin rejettent cette image archétype. Calvin, grand humaniste lui-même, part aussi de la philosophie chrétienne mais pour aboutir à d'autres conclusions il n'y a pas "de liberté en la volonté de l'homme". L'homme ne peut soutenir aucun combat par lui-même car il est l'esclave du péché et de la corruption. L'ennemi est déjà dans la place. Créé à l'image de Dieu, l'homme ne recouvre cette dignité que s'il connaît d'abord Dieu par la vénération de l'image du Christ qui est en lui-même. Calvin meurt en 1564, année de la naissance de Shakespeare. Montaigne a trente et un ans. Tous deux appartiennent à la seconde moitié du Xvle siècle, c'est-à-dire au moment où les tensions induites par l'inflexion dont parle 0 . Boulnois atteignent leur paroxysme. Nous soutiendrons que leurs oeuvres reprennent les débats qui les ont précédées en ce qu'elles examinent, elles aussi, les rapports entre Dieu, l'homme et le monde. Nous démontrerons en quoi Montaigne et Shakespeare diffèrent conjointement des auteurs de la première moitié de leur siècle et comment, s'il est possible de parler d'un nouvel humanisme, ce dernier est radicalement étranger à ce qui, aujourd'hui, justifie la notion de dignité humaine.

"I must be cruel only to be kind". The concept of human dignity in Montaigne and Shakespeare Humanism, according to Olivier Boulnois, marks the moment when the medieval thinking on the relationships between God, man and the world shifted to a new, modern one. From what was then called Christian philosophy, Pico de La Mirandola and Erasmus, to name only those two thinkers, deduced the argument of man's fundamentally dignified nature. Man, thanks to the gift of free will he received from God, can transcend his animal nature and then recover his divine essence through epopteia or, in the words of Petrarch, he can move from the state of homo to that of vir. Human dignity comes into being through the force (virtù) and the virtue necessary for man to fight off the forces of Evil that are for ever assailing his station. Opposed to this archetypal image were the views of religious Reformers and, more particularly, of Jean Calvin. The latter was a great humanist himself, but the conclusions he drew from what he also called Christian philosophy were radically different: there is "no liberty in the will of man". There is no fight man can sustain on his own as he is a slave to sin and corruption. Indeed, the enemy is already within the walls. It is true man was created in the image of God, but this dignity can be recovered only if he first knows God through the veneration of the image of Christ which is in himself. Calvin died in 1564, the year Shakespeare was born. Montaigne was then thirty one. Both Montaigne and Shakespeare belong to the latter part of the $16^{\text {th }}$ century that is when the tensions due to the shift 0 . Boulnois speaks of were becoming paroxysmal. I shall first argue that the works of Montaigne and Shakespeare must be viewed in the continuity of the debates that preceded them as they indeed also question the relationships between God, man and the world. Secondly, I shall examine in what ways they jointly differ from the humanists and the Reformers of the first half of the $16^{\text {th }}$ century. Finally, I shall show that if one may speak of a new kind of humanism as regards their views, it is nonetheless radically different from what, today, vindicates the notion of 'human dignity'.
\end{abstract}


livier Boulnois définit l'humanisme comme le point d'inflexion entre la pensée médiévale et la pensée moderne des rapports entre Dieu, l'homme et le monde ${ }^{67}$. Pour évaluer l'évolution de Montaigne et de Shakespeare vers un nouvel humanisme, nous prendrons trois points de repères: Pic de La Mirandole, Érasme et Calvin. Ils forment deux groupes, d'un côté Pic et Érasme, de l'autre Calvin. Enfin, nous partirons d'une phrase surprenante, tirée de l'essai « De la cruauté » (II, 11) : « Nature, à ce creins-je, elle-mesme attache à l'homme quelque instinct à l'inhumanité ${ }^{68}$. Pour Shakespeare, nous retiendrons ce que dit Hamlet et qui semble faire écho à Montaigne : «I must be cruel only to be kind» (IV.iii. 180 $)^{69}$.

Alexandre Dumas, en 1866, le premier il semble, a traduit «kind» par «humanité» dans l'acception courante de «bienveillance» ${ }^{70}$. À la même époque, Robert Villiers appuie davantage cette interprétation ${ }^{71}$. Avant Dumas, «kind» est traduit par « humain ». C'est le cas, au XVIII siècle, chez Le Tourneur ${ }^{72}$. En 1859, François-Victor Hugo traduit «kind» de la même façon, mais marque plus nettement le sens restrictif de «only»: «Il faut que sois cruel, rien que pour être humain ${ }^{73}$.» Toute l'ambiguïté de l'énoncé provient de l'interprétation du mot «kind» et de la portée de l'adverbe «only». «Kind» peut être soit adjectif, soit nom commun. Il vient de l'anglo-saxon cynd rattaché lui-même à $\operatorname{cin}$ qui a donné kin, la famille. Shakespeare a exploité l'association entre kin et kind dans la toute première réplique d'Hamlet

\footnotetext{
67 Olivier Boulnois, «Humanisme et dignité de l'homme selon Pic de La Mirandole», in Jean Pic de La Mirandole, Euvres Philosophiques, texte latin, trad. et notes O. Boulnois et Giuseppe Tognon, coll. Epimethée, Paris, PUF, 1993, p. 294.

68 Montaigne, Les Essais, éd. Pierre Villey, préf. V.-L. Saulnier, coll. Quadrige, Paris, PUF, 1988, p. 433. Les citations tirées de cette édition seront notées dans le texte.

${ }^{69}$ Shakespeare, Hamlet, éd. Harold Jenkins, coll. The Arden Shakespeare, Londres, Methuen, 1981, p. 330

70 Shakespeare, Ham let, trad. Alexandre Dumas, in Le Siècle, 1866.

${ }^{71}$ Shakespeare, Hamlet, trad. Robert Villiers, coll. Bibliothèque Universelle de Poche, Fayard, Paris, s.d.

72 Shakespeare traduit de l'anglois par M. Le Tourneur, dédié au Roi, tome V, s.l. 1779,

73 Cuvres complètes de Shakespeare, tome I, Les Deux Hamlet, le «Second Hamlet», Paris, Pagnere, 1859, p. 273.
} 
(I.ii.64-65). Après consultation du dictionnaire d'Anglo-Saxon ${ }^{74}, \mathrm{du}$ Dictionnaire de Moyen Anglais ${ }^{75}$, du Oxford English Dictionary il apparaît, qu'il est indifférent que kind soit adjectif ou nom. Kind doit s'entendre dans son sens originel, c'est-à-dire ce qui est ou renvoie à la nature, qu'il s'agisse d'ordre naturel ou de nature individuelle. Nous soutiendrons que kind signifie ici «de l'espèce humaine, de la nature humaine ou encore du genre humain » et ne renvoie pas à la notion de bienveillance qui ne se trouve que parmi les acceptions de l'adjectif. Un premier sens se dégage : «Je dois être cruel seulement pour être du genre humain. » L'adverbe only pose aussi un problème. En raison de la césure, il appartient à la deuxième partie du vers ${ }^{76}$. Néanmoins, la restriction porte-t-elle sur cruel ou sur kind? Only se trouve au milieu du vers et renforce ainsi l'ambiguïté de l'énoncé. Les traducteurs modernes font porter only sur cruel. C'est le cas de Jean-Michel Déprats $^{77}$, de Michel Grivelet ${ }^{78}$ et de Jean Malaplate ${ }^{79}$. La portée de l'adjectif cruel s'en trouve diminuée car elle renvoie alors à une disposition psychologique passagère. Or, l'adjectif anglais cruel est un emprunt direct du français cruel qui, lui-même, vient du latin crudelis relié à crudus c'est-à-dire cru. Ce dernier a une racine indo-européenne qui exprime les notions de «chair crue» et de «sang répandu», représentées par le sanskrit kravyan pour «chair crue» et le grec kreas, kreatos pour «chair saignante». Donc, cruel ne qualifie pas une disposition psychologique mais un état, ici une façon d'être. La valeur morale que l'on trouve dans la restriction de l'adjectif cruel avec l'adverbe only n'a alors pas de sens parce qu'il s'agit, dans le vers prononcé par Hamlet, d'un constat objectif et non d'une évaluation subjective et morale. L'adverbe only porte bien sur kind. Enfin, le modal must n'indique pas l'obligation morale mais plutôt la

\footnotetext{
${ }^{74}$ Anglo-Saxon Dictionary, based on the manuscript collections of the late Joseph Bosworth, éd. T. Northcote Toller, Londres, Oxford University Press, 1972, p. 183.

${ }^{75}$ Middle English Dictionary, éd. Sherman M. Kuhn et John Reidy, Ann Arbor, University of Michigan Press, 1969, p. 500

76 Je remercie Jean-Marie Maguin pour cette précision.

77 Shakespeare, Ham let, III.iv.177, texte établi par Henri Suhamy, trad. J.-M. Déprats, coll. La Pléiade, Paris, Gallimard, 2002, tome I, p. 871.

78 William Shakespeare, Euvres Complètes, éd. bilingue, Tragédies, vol. I, éd. Michel Grivelet et Gilles Monsarrat. Ham let, III.iv.162, trad. et notes M. Grivelet, coll. Bouquins, Paris, Robert Laffont, 1995, p. 990.

79 Shakespeare, Ham let, III.iv.164, Paris, José Corti, 1991, p. 174.
} 
contrainte: I have to. André Gide l'a interprété de cette façon ${ }^{80}$. Nous aboutissons au sens suivant : "I have to be cruel to be only human"; où «humain » signifie « espèce humaine », «human kind ». Celle-ci constitue un état premier, cruel au sens étymologique du terme, et insuffisant pour fonder la dignité de l'homme.

Dan s La Vie Solitaire, Pétrarque distingue entre un état premier de l'homme, celui de l'animalité, qu'il désigne avec homo ; et un second état, celui de l'humanité, désigné par humanitate. Il faut se dépouiller de sa férocité pour se vêtir de l'humanité et devenir "homme», vir. Christophe Carraud traduit ainsi: « un animal dangereux, instable, déloyal, perfide, cruel et sanguinaire, - tel est l'homme. À moins qu'il n'ait appris [...] à se dépouiller de l'animalité pour se revêtir de l'humanité, - bref, de simple humain qu'il était, à devenir vraiment homme ${ }^{81}$. Carraud précise que la traduction «humain / homme» peine à rendre justice à la juxtaposition d'homo et de vir. Ce dernier mot, d'après Cicéron, constitue pour ainsi dire la substance première de la vertu, la virtus (Cicéron, Tusculanes, 2, 18, 43) qui dérive étymologiquement de lui. Cicéron dit exactement : "Vertu a la même racine que viril. Or ce qui caractérise essentiellement une âme virile, c'est le courage, et le courage a deux attributions essentielles qui sont le mépris de la douleur et le mépris de la mort. Il faut donc pratiquer ce mépris si nous voulons être vertueux ou mieux si nous voulons être des hommes, puisqu'aussi bien vertu vient de viril ${ }^{82}$. »

Le rapprochement de vir et virtus indique que l'homme porte en lui le dépassement de sa nature animale. Chez les deux auteurs, la transformation de l'homme animal aboutit à une forme supérieure, vertueuse de l'homme plus proche d'une forme d'essence divine. La différence entre eux tient dans la façon dont l'homme s'accomplit en lui-même et par lui-même. Chez Cicéron c'est au moyen du courage ; ce qui renvoie à l'image du combattant, image reprise par Érasme. Chez Pétrarque, l'accomplissement de l'humanité se fait par l'étude des humanitas, ces disciplines plus humaines (humaniores disciplinae) qui s'opposent au formalisme de la scolastique médiévale. Ces deux

${ }^{80}$ Shakespeare, Ham let, trad. André Gide, coll. La Pléiade, Paris, Gallimard, tome II, 1959, p. 669.

${ }^{81}$ Pétrarque, Préface, 9 ; in La Vie solitaire, éd. Christophe Carraud, Grenoble, Jérôme Million, 1999, p. 35.

82 Cicéron, Tusculanes, II, xviii, 44, texte établi par G. Fohlen, trad. J. Humbert, éd. revue et corrigée par Claude Rambaux, Paris, Les Belles Lettres, 1997, p. 101. 
attitudes ne s'excluent pas. Dans l'anthropologie médiévale l'homme représente le lieu où se noue le drame entre sa nature déchue, ancrée dans la matérialité, et réalité objective; et l'image de Dieu qu'il représente, réalité abstraite. La résolution de cette tension réside dans la vertu. La vertu est alors la voie par laquelle l'homme s'humanise.

Montaigne aussi se place dans cette tradition qui veut que l'homme n'est véritablement homme que par accomplissement de luimême. Une part importante de l'essai « De la cruauté » (II, 11) traite de la vertu. L'objet de l'essai n'est pas de fournir la description d'actes de cruauté comme chez Las Casas dans La Destruction des Indes $(1552)^{83}$, ou chez Verstegan dans Le Théâtre des Cruautés (1587) ${ }^{84}$. Montaigne s'interroge sur l'origine même de la cruauté. Traditionnellement, l'homme cruel a perdu sa véritable nature. De ce fait, la cruauté est étrangère à la nature humaine. Montaigne inverse cette conception : «Nature, à ce creins-je, elle mesme attache à l'homme quelque instinct à l'inhumanité» (II, 11, 433b). Cette phrase a été retouchée de nombreuses fois. Dans l'édition de 1595, chez Sonnius à Paris, le présent a été remplacé par un passé composé et «ce crains-je » est mis entre parenthèses ${ }^{85}$. Une modification identique se trouve dans l'édition publiée par F. Le Febure, à Lyon, toujours en 1595. La présence de l'auxiliaire «avoir» est clairement indiquée à l'aide de virgules qui l'encadrent ${ }^{86}$. En 1600, chez Abel L'Angelier, et toujours sous l'autorité de Mademoiselle de Gournay, le passé composé a été conservé et le mot inhumanité a été remplacé par humanité : "Nature a, (ce crains-je) elle mesme attaché à l'homme quelque instinct à l'humanité ${ }^{87}$. Il faut voir dans cette modification l'avènement de la conceptualisation moderne de l'homme. Avec l'essor des sciences, il

83 Bartolomé de Las Casas, La Destruction des Indes (1552), trad. Jacques de Miggrode (1579), gravures de Théodore de Bry (1598), introduction d'Alain Milhou, analyse iconographique de J.-P. Duviols, Paris, Chandeigne, 1995

${ }^{84}$ Verstegan, Richard, Théâtre des cruautés des hérétiques de notre temps, éd. Frank Lestringant, Paris, Chandeigne, 1995.

${ }^{85}$ Les ESSAIS de Michel Seigneur de Montaigne. Edition nouvelle trouvée apres le deceds de l'Autheur, revue et augmentée par luy d'un tiers plus qu'aux precedentes impressions. Imprimé par M. Sonnius à Paris. 1595, p. 278.

${ }^{86}$ Les Essais de Michel Seigneur de Montaigne, divisez en trois livres contenans un riche et rare thresor de plusieurs beaux et notables discours couchez en un stile le plus pur et orné qu'il se trouve en nostre siecle. [...] Imprimé par F. Le Febure éditeur à Lyon, 1595, p. 380.

${ }^{87}$ Les Essais de Michel Seigneur de Montaigne. Edition nouvelle, prise sur l'exemplaire trouvé parès le deceds de l'Autheur, revu et augemnté d'un tiers outre les precedentes impressions. Chez Abel L'Angelier, Paris, 1600, Livre II, p. 426. 
devient inacceptable de dire que la nature de l'homme est naturellement et d'instinct inhumaine. Shakespeare se trouve au confluent de ces deux conceptions.

Florio, dans sa traduction des Essais, publiée en 1603 à Londres en un volume in-folio compact, garde le passé composé et le mot humanité, ce qui donne: "Nature (I feare me) hath of hir owne selfe added vnto man a certaine instinct to humanity ${ }^{88}$. Florio utilise aussi le verbe « ajouter» et non «attacher», ce qui fait de la nature humaine une entité à laquelle s'ajoute un élément indépendant. Il rejoint alors la position traditionnelle. D'autre part, Florio rend encore plus évident le caractère absurde de la modification apportée au texte des Essais car Montaigne dirait alors qu'il craint que la Nature ait ajouté à la nature humaine un instinct à l'humanité. En quelque sorte, Florio se trahit. Il veut éviter le mot inhumanité rattaché à la nature humaine. Ce qui est en débat, c'est la nature de l'homme.

Dans la phrase «Nature, à ce creins je...» le mot «nature» représente à la fois la Nature créée et la nature individuelle de chaque homme. Montaigne fonde cette proximité premièrement par rapprochements successifs d'abord des bêtes à l'homme, puis des arbres et des plantes : "Nous devons la justice aux hommes, et la grace et la benignité aux autres créatures qui en peuvent estre capables. Il y a quelque commerce entre elles et nous, et quelque obligation mutuelle » (II, 11, 435). Dans ce passage se trouve par deux fois la notion de devoir. Relevons aussi le mot "humanité». Au $\mathrm{XVI}^{\mathrm{e}}$ siècle il signifie : «urbanité, affabilité, bienveillance ${ }^{89}$. Montaigne le relie implicitement aux mots de justice, grâce et bénignité qui, tous trois, renvoient à la notion de divinité. Il élargit le sens du mot humanité par association à des attributs divins et non à ce qui émanerait de la simple nature de l'homme. Le mot «commerce» souligne la similitude de condition de tous les étants. Plus haut, Montaigne s'appuie sur la théologie pour suggérer une condition similaire entre les hommes et les animaux par le biais de l'appartenance à une même famille, celle de

88 The Essayes or Morall, politike and Militarie Discourses of Lo: Michael de Montaigne, Knight of the noble Order of St Michael, and one of the Gentlemen in ordinary of the French King, Henry the Third his Chamber. First written by him in French, an now done into English by [...] John Florio. Printed at London by Val. Sims for Edward Blount dwelling in Paules Churchyard, 1603, p. 250

${ }^{89}$ Huguet, Edmond, Dictionnaire de la langue française du $16^{e}$ siècle, 7 tomes, Paris, Didier, 1925-1967. 
Dieu (II, 11, 433). En définitive, ce qui est commun c'est la condition. Quant à la nature individuelle, elle est due au sort et à rien d'autre. La propre nature de Montaigne illustre ce point (II, 11, 429). Il n'y a pas d'action extérieure qui puisse agir sur la nature individuelle. Or, la métaphore du combat était fondatrice de la vertu et donc de la dignité humaine. Montaigne fait référence à cette tradition au début de son essai (II, 11, 422), mais cet argument est une fausse piste (II, 11, 423). À la conception traditionnelle de la vertu Montaigne oppose l'exemple de Socrate : «Au train de sa vertu, je n'y puis imaginer aucune difficulté et aucune contrainte»(II, 11, 423a). Montaigne tourne ainsi le dos à l'héritage stoïcien et à l'enseignement de l'Église issu de cette même tradition. Plus bas dans l'essai, il indique clairement qu'il préfère la vertu telle que Socrate la pratique : "Or qu'il ne soit plus beau, par une haute et divine resolution, d'empescher la naissance des tentations, et de s'estre formé à la vertu de maniere que les semences mesmes des vices en soyent desracinées [...] je ne pense point qu'il y ait doubte (II, 11, 426a). «Resolution », ici, connote le dénouement, l'ouverture, en raison du latin resolutio, action de dénouer. Le sens du mot résolution est explicité plus bas dans l'essai avec les exemples de Socrate et de Stilpo qui ont apprit à connaître leurs vices pour se dégager de leur emprise (II, 11, 429). La vertu ne consiste plus à lutter contre un ennemi extérieur que représentent les vices et les tentations car ceux-ci n'existent pas en dehors de l'homme. L'ennemi se trouve déjà dans les murs et il est consubstantiel à l'individu lui-même; thème que l'on retrouve notamment dans les tragédies de Shakespeare. Montaigne abandonne la métaphore de la place forte assiégée et donne à l'injonction socratique du «connais-toi toi-même» le sens moral que ses contemporains lui déniaient. La connaissance de soi devient ainsi le moyen d'accès à la vertu. L'individu devient ainsi l'artisan de son propre salut; ce qui fait référence au libre arbitre et au problème de la justification. Ces deux points étaient au cœur de nombreuses controverses au XVI ${ }^{\mathrm{e}}$ siècle. Cependant, Montaigne reste dans un cadre religieux. En effet, nous avons vu que la connaissance de soi conduit à une haute et divine résolution; ce qui nous renvoie à l'image de l'homme temple de Dieu qui se trouve chez saint Paul (1 Corinthiens, 3, 16) $)^{90}$. D'autre part le mot «résolution» dans le sens de

90 La Bible, Traduction œcuménique, Paris, Édition du Cerf / Villiers-le-Bel, Société Biblique Française, 1997, p. 2749. 
«dénouement» connote un passage important chez saint Paul dans l'Épître aux Romains: «7, 14: Nous savons, certes, que la loi est spirituelle ; mais moi, je suis charnel, vendu comme esclave au péché. [...] 7, 24: Malheureux homme que je suis! Qui me délivrera de ce corps qui appartient à la mort ? ${ }^{91}$ Pour saint Paul la délivrance se fait par la foi dans le Christ; thème que reprendra Calvin. Chez Montaigne cette délivrance est tout autant spirituelle mais laïque dans la mesure où elle repose sur le libre arbitre des individus et le devoir de se connaître. Cette conception de l'homme est proche de celle de Pic de La Mirandole mais pourtant différente.

Dans son Discours sur la dignité de l'homme (1486, publié en 1504), Pic de La Mirandole voit dans le libre arbitre, don de Dieu, le seul élément véritablement fondateur de la dignité de l'homme. Il reprend le mythe d'Épiméthée. Après avoir terminé la Création, Dieu souhaite donner vie à une créature capable d'admirer son œuvre. Mais tous les archétypes ont été utilisés. Pic prête alors à Dieu les paroles suivantes: «Je ne t'ai donné ni place déterminée, ni visage propre, ni don particulier, ô Adam, afin que ta place, ton visage et tes dons, tu les veuilles, les conquières et les possèdes par toi-même. [...] Tu pourras dégénéré en formes inférieures, comme celles des bêtes, ou, régénéré, atteindre les formes supérieures, qui sont divines ${ }^{92}$. $\gg$ Pic s'appuie sur la tradition qui fait de l'homme un être à mi-chemin entre le monde des bêtes et le monde des esprits. Cependant, pour lui, il est donné à l'homme de choisir ce qu'il désire être (p. 7). En raison du libre arbitre, l'homme est donc seul responsable de son devenir. En effet, le libre arbitre est bon en soi mais l'homme peut le corrompre (p. 13). Pic met l'homme en garde: «Qu'une sainte ambition envahisse notre esprit pour que, sans nous contenter des choses médiocres, nous aspirions aux plus hautes et appliquions toutes nos forces à les atteindre puisque, du moment que nous voulons, nous pouvons» (p. 13). La vertu, chez Pic, reste dans la tradition stoïcienne. Elle est une philosophie de l'opposition, du combat.

Érasme, se situe dans le même courant. L'accès à la vertu nécessite force et courage. C'est le thème de l'Enchiridion Militis Christiani (1504 et 1518) : "Tout d'abord, il te faut rappeler sans cesse

91 Ibid., p. 2716

92 Pic de La Mirandole, Euvres Philosophiques, op. cit., p. 5. Toutes les citations seront de cette édition. 
que la vie des mortels n'est rien d'autre qu'une sorte de service militaire perpétuel ${ }^{93}$. Le danger vient du monde visible comme du monde invisible. Érasme exploite le récit biblique de la chute de l'homme. Le danger est ainsi conçu sous la forme du poison, moyen privilégié pour corrompre l'esprit de l'homme (p. 90). Le danger est partout car le monde entier est plongé dans le mal (p.90). Galilée n'est pas encore né. Le modèle géocentrique de la création reste la référence. Érasme l'exploite ici sur le plan moral. En haut se trouve un monde supérieur. La terre est «en bas ». Tous les déchets de la Création s'y concentrent comme le souligne aussi Pic. Dans ce monde, le serpent réside toujours, poursuivant obstinément les mêmes fins (p. 90). Ces éléments se retrouvent chez Shakespeare dans le récit que fait le fantôme (I.v.34-40). Se retrouve ici l'opposition classique entre un monde spirituel, supérieur, et un monde matériel, inférieur. Érasme transpose ensuite cette opposition chez l'homme où il y a aussi deux régions. L'une est sa spiritualité. Elle correspond à sa masculinité. La seconde est celle de sa matérialité ; la partie féminine de sa nature. Cette dernière représente la porte d'accès par laquelle le Mal corrompt la spiritualité de l'homme: «Par «femme», entends la partie charnelle de l'homme. C'est là notre Ève, par laquelle ce très rusé serpent attire notre esprit vers les voluptés mortifères» (p.90). Nous retrouvons ici, dans une perspective chrétienne, l'héritage de Cicéron. L'homme véritable est l'homme dépouillé de la partie charnelle, féminine et faible de sa constitution. D'autre part, le texte d'Érasme nous renvoie à ce que dit Hamlet: «Frailty, thy name is woman » (I.i.146). Enfin, Érasme opère une troisième et dernière division au sein même de l'esprit de l'homme entre le Vieil Adam et le Nouvel Adam. Cette distinction est introduite par saint Paul dans l'Épître aux Colossiens: (3, 5-10, TOB, Cerf, p. 2859). Érasme la reprend et la dynamise dans la tradition allégorique initiée par Prudence dans sa Psychomachia ${ }^{94}$. Le Vieil Adam continue de résider au sein même de l'esprit du Nouvel Adam, l'homme nouveau régénéré par le sacrifice du Christ : «nous portons au dedans de nous, dans le plus intime de notre esprit, un ennemi plus que familier, [...] C'est là, oui, ce vieil et terrestre

\footnotetext{
93 Érasme, Enchiridion Militis Christiani, trad. A. J. Festugière, Paris, Vrin, 1971, p. 89. Toutes les citations de cette œuvre renverront à cette édition .

94 Prudence, Psychomachie, trad. Maurice Lavarenne, revue par Jean-Louis Charlet, Paris, Les Belles Lettres, 2002.
} 
Adam, par l'accoutumance plus que notre concitoyen, par ses penchants plus que notre ennemi [...]. Nous devons l'épier, de peur qu'il n'ouvre aux démons le poste fortifié de Dieu» (p. 90). Avec cette dernière formulation, nous retrouvons la notion de station de l'homme. Elle se trouve chez Platon dans le Phédon (62) et aussi chez Sénèque, dans les Lettres à Lucilius (VI, 56, 9, Bouquins, Flammarion, p. 730). Elle jouit d'une grande popularité pendant tout le Moyen-Âge. Calvin l'a reprise dans l'Institution de la Religion Chrétienne au livre III et son argumentation contre le suicide suit exactement celle du Phédon ${ }^{95}$ Chez Érasme cette image fait de l'homme un soldat chargé de défendre le poste qu'il a reçu de Dieu, à savoir le corps qui retient son âme (p. 91). Ainsi l'ennemi est-il partout et la guerre permanente.

Érasme fonde son anthropologie sur un système d'oppositions allégoriques dont il indique la portée pédagogique (p. 145) Ces pages sur l'allégorie aboutissent à l'opposition entre les théologiens et les humanistes, et à la reprise de l'opposition classique, entre l'esprit et la lettre. Mais avant cela, Érasme souligne que l'allégorie fut introduite par le Christ lui-même, suivi par l'apôtre Paul et les Pères de l'Église (p. 146). Érasme place donc l'esprit vivifiant du côté des humanistes et des auteurs classiques païens parce que les uns comme les autres se retrouvent dans la tradition établie par le Christ lui-même. La théologie vivante est donc celle des humanistes. L'allégorie a donc pour objet de rendre compte d'une réalité complexe, et comme chez Pic, elle joue un rôle important chez Érasme. Le système d'oppositions sert aussi pour décrire la nature de l'homme, créature hybride: «l'homme est une sorte d'animal prodigieux composé de deux ou trois parties tout à fait diverses» (p. 109). Il poursuit pour dire que, par les qualités corporelles, la bête l'emporte sur l'homme (p. 109). Cela, Montaigne le conteste et il ne reprend pas l'opposition entre nature animale (le corps) et nature divine (l'âme). L'enjeu est ailleurs. Chez Érasme, ces deux natures co-existent de façon objective. Ainsi, grâce à cette nature divine l'homme se trouve toujours en rapport direct avec Dieu (p. 109) Érasme aboutit à l'image d'une guerre généralisée à l'intérieur comme à l'extérieur de l'homme; ce qui correspond aux aspirations contradictoires de l'homme, extérieures par les besoins du corps, intérieures par les besoins de l'âme. Érasme pose la question de la

95 Jean Calvin, Institution de la Religion Chrétienne (1560), éd. Jean-Daniel Benoit, Paris, Vrin, 1960, III, ix, 4, p. 193 
responsabilité de cet état de chose. Avant la Chute, ces deux natures étaient ordonnées et hiérarchisées ; mais le péché a mis fin à cette harmonie (p. 109). La responsabilité de l'homme se trouve relativisée puisqu'il est la victime d'une force concrète et maligne qui a tout corrompu. Cependant, les conséquences de cette corruption aboutissent à une catastrophe au sens propre du terme car désormais : « les passions du corps font assaut pour dominer la raison, et celle-ci est forcée de se ranger à l'avis du corps » (p. 110). Érasme poursuit avec la métaphore du corps comme organisation politique et fait référence à Platon dans le Timée (p. 110-111), où il trouve confirmation de l'existence d'une nature double chez l'homme (p. 112). Chez Montaigne, la difficulté pour appréhender l'homme n'est pas due à la nature humaine qui serait double mais plutôt au fait de la diversité des natures dans l'espèce humaine. Car il n'y a pas une nature chez l'homme, simple ou composée, mais des natures individuelles, bonnes, indifférentes ou mauvaises. La même notion se trouve chez Shakespeare dans Macbeth (III.i.92-103). Érasme trouve ainsi chez Platon confirmation de sa conception de la vertu (p. 111) Le thème de la vigilance réapparaît ici. Montaigne utilise lui aussi cette notion mais envers soi, envers cet inconnu qui est soi-même. La vigilance doit s'appliquer envers la nature individuelle que l'on a reçue, de façon mystérieuse, à la naissance. Parlant de lui-même, Montaigne écrit dans l'essai «De la cruauté»: «Les desbordemens ausquels je me suis trouvé engagé, ne sont pas, Dieu mercy, des pires. Je les ay bien [...] retranchez et contrains les plus seuls et les plus simples que j'ay peu » (II, 11, 428a). Avec les verbes «retrancher» et «contraindre», nous retrouvons les métaphores guerrières de l'Enchiridion. Cependant, la lutte ne concerne pas un ennemi extérieur mais les différentes passions qui constituent soi-même.

Érasme, enfin, avec la citation de Platon aboutit aussi à la fusion des concepts de divinité, de raison et de royauté (p. 111). Cette description renvoie à la division générale de la Création divine en parties inférieure et supérieure, division appliquée à l'organisation physiologique de l'homme et représentée par le diaphragme qui sépare la tête, l'acropole, de «la partie concupiscente» (p. 112). La ligne de partage représentée par le diaphragme chez l'homme s'applique aussi au corps politique pour distinguer entre le palais royal, siège de la raison et l'écurie où logent les passions, c'est-à-dire le peuple (p. 112). 
Un troisième élément relie ces deux extrêmes, c'est le cœur, siège à la fois du courage et de la colère (p. 112). Chez Montaigne, il n'y a plus de correspondances allégoriques entre le corps humain et l'État. D'autre part, il inverse le rapport traditionnel entre raison et passions : «je trouve par là, en plusieurs choses, plus d'arrest et de reigle en mes meurs qu'en mon opinion, et ma concupiscence moins débauchée que ma raison » (II, 11, 428b). Avec la reprise des mots concupiscence et raison, Montaigne se place bien dans la tradition des débats qui les concernent; mais il suggère que la raison est aussi une passion et, de plus, la plus violente. Le concept de vertu s'en trouve profondément modifié. Il ne s'agit plus de désapprendre le mal. Pour Montaigne, avec une telle image on se paie de mots (II, 11, 428). La vertu est une préoccupation individuelle et non un ensemble de préceptes généraux. Est vertueux celui qui apprend à se connaître de façon à reconnaître et à contrôler les passions dont il est fait. Montaigne a compris toute la portée morale du précepte delphique et socratique. Florio a correctement traduit ce passage : «I therby find in many things, more stay and order in my maners, then in my opinion: and my concupiscence lesse debauched, then my reason»(p. 247). Les deux mots clefs, concupiscence et raison constituaient des marqueurs facilement reconnaissables. Florio les a réemployés d'autant plus aisément que l'inversion du rapport habituel qui est ainsi opérée correspond à l'image que Calvin donne de la raison humaine, à savoir une raison débilitée par le péché (IRC, II, ii, 12, p. 36). Calvin avait conservé la distinction entre raison et intelligence (IRC, I, xv, 6, p. 216). Montaigne la radicalise. La raison c'est, selon l'étymologie ratio, le calcul. L'intelligence, en revanche, c'est ce qui relie les hommes entre eux et les hommes aux dieux. Shakespeare montre aussi comment la raison est mise au service des passions; par exemple, dans Titus Andronicus, lorsque Titus justifie le sacrifice d'Alarbus (I.i).

La raison, cependant, chez Érasme, c'est le moyen d'accès à la vertu. Pourtant, il manifeste la conscience d'un détournement de la raison au profit des passions (Enchiridion, p. 115). Érasme fait preuve de cohérence, puisque auparavant il décrit la catastrophe qui, par le péché, a corrompu l'ordre hiérarchique entre la raison et les passions. La voie du salut ne se trouve que dans le Christ. Comment Le rejoindre ? Érasme utilise aussi le précepte socratique mais dans une perspective strictement chrétienne. Érasme fusionne l'héritage 
classique et l'enseignement biblique; mais il conserve le principe de division binaire pour distinguer entre la fausse et la vraie sagesse (p. 106). La vertu est conçue comme un but qui se trouverait devant soi. Ce but se confond avec Dieu qui est « le père et l'auteur de toutes les vertus». Il n'en est pas de même chez Montaigne. Au début de l'essai « De la cruauté », il s'appuie sur la conception classique de la vertu en tant qu'opposition au déraisonnable pour dissocier Dieu du concept de vertu: «car il semble que la vertu presuppose de la difficulté et du contraste, et qu'elle ne peut s'exercer sans partie. C'est à l'aventure pourquoy nous nommons Dieu bon, fort, et liberal, et juste ; mais nous ne le nommons pas vertueux : ses opérations sont toutes naïves et sans effort» (II, 11, 422a). Ici s'exprime un autre renversement. En effet, Dieu n'est plus concerné par le concept de vertu; seuls les hommes le sont. Cela implique d'importantes conséquences liées à la nature de l'homme, à sa responsabilité et au libre arbitre. Montaigne replace le problème de la vertu au sein même du monde. Voici un exemple précis de l'inflexion dont parle Olivier Boulnois entre la pensée médiévale et la pensée moderne des rapports entre Dieu, l'homme et le monde. La poésie remplace l'allégorie pour rendre compte de la complexité du monde. Par sa pratique théâtrale et poétique, Shakespeare illustre cette inflexion.

Érasme est encore ancré dans la tradition médiévale. Le système binaire qu'il utilise implique non seulement des forces contraires mais aussi des renversements de nature apparente. C'est le cas pour la sagesse. Il suit saint Paul pour lequel la sagesse de ce monde est folie aux yeux de Dieu et inversement. Dans cette perspective, le précepte delphique et socratique prend, lui aussi, deux sens opposés. Premièrement, la connaissance de soi pour soi représente l'expression de l'orgueil humain. La faiblesse de l'homme est trop grande, la confusion dans laquelle il se trouve est trop radicale pour qu'il puisse prétendre pouvoir agir pour son salut. Nous l'avons déjà vu chez saint Paul dans l'Épître aux Romains (chap.7). Érasme reprend ce thème : "Cesse de t'appuyer sur toi-même, jette-toi en lui [Jésus]» (Enchiridion, p. 131). Érasme condamne l'amour de soi parce c'est ainsi aimer le vide (p. 163). Enfin, tout ce sur quoi l'homme fonde sa dignité lui vient de Dieu. La connaissance de soi consiste alors à reconnaître cette dette (p. 209). Se connaître, alors, c'est admettre que l'on est rien (p. 210). Le deuxième sens concerne l'état spirituel de 
l'homme. Cet état, Érasme le décrit comme un ensemble de vices et d’iniquité. La connaissance de cet état s’identifie à la sagesse ; mais cette dernière reste l'expression de la grâce de Dieu. Un homme aussi spirituel que saint Paul n'osait se juger lui-même, faute de se connaître assez. Donc, le simple mortel ne peut prétendre qu'à une connaissance toute relative de lui-même. Cette connaissance revêt, en fait, un aspect pratique. C'est celle qui est nécessaire au soldat pour combattre efficacement. En définitive, la connaissance de soi consiste à distinguer ce qui est ami et ce qui est ennemi en soi-même (p. 108). Érasme explique le précepte delphique dans le cadre de l'allégorie de la place forte assiégée, de la station avec des forces extérieures et des forces intérieures mais, maintenant, transposée au sein même de l'homme. Le but de la connaissance de soi est donc la victoire sur soi. Il ne s'agit pas ici d'une connaissance subjective du sujet mais objective. Érasme entreprend ensuite de montrer à l'homme ce qu'il est (p. 108). Il suit donc la tradition selon laquelle la vertu consiste à fuir le mal et à rechercher le bien à l'aide de la raison, et il relie cette tradition à Socrate (p. 166). Or, la raison occupe la position du roi dans la république que représente le corps humain. Ce corps humain fait partie du corps représenté par l'ensemble des hommes et la tête de ce corps est le Christ (p. 178) La raison s’identifie donc à Jésus, et, par suite, à Dieu le Père. La justesse de l'héritage classique a été révélée par le Christ mais ce dernier reste le modèle, l'archétype (p. 167). Érasme distingue donc entre l'état de péché et l'état de dignité humaine. Ce qu'il faut fuir, c'est le péché. Se connaître soi-même c'est comprendre la différence entre l'état ignoble du péché et la grandeur de la dignité humaine. Cette dignité n'est pas fondée en l'homme même, comme chez Pic de La Mirandole, mais en Dieu, parce que l'homme est membre du Christ et image de la divinité. Pour Érasme, l'homme doit fuir l'état de péché pour retrouver l'état de dignité humaine. Se connaître soi-même, c'est reconnaître l'existence de ce choix entre l'indignité et la dignité humaine. Avec le précepte delphique, Érasme érige deux états moraux compartimentés qui s'excluent mutuellement (p. 197). Ces deux états, péché et dignité humaine, appartiennent à l'allégorie. Ils ont une existence propre. Dans ce cadre de pensée, il est possible de passer de l'un à l'autre. Ce geste relève alors de la délibération fondée sur la raison. Ici intervient donc le libre arbitre. 
Pic de La Mirandole fait aussi référence à Socrate pour opposer les lieux du péché et de la divinité (Discours, p. 29). Mais il donne, plus bas dans le Discours, une autre interprétation du «connais-toi toimême » qui est radicalement différente de celle d’Érasme. En effet, se connaître, pour Pic, c'est accéder à la connaissance de la Nature entière, donc de la Création dans son ensemble et, par voie de conséquence, c'est accéder à la connaissance de l'homme et de Dieu parce que tout est compris en la Nature (Discours, p. 31). Pic oppose donc un état d'ignorance à l'état de connaissance de la Nature, état qui se confond avec la sagesse. Cependant, il ne s'agit pas de fuir un état pour en rejoindre un autre, comme chez Érasme. Il s'agit de purifier un état qui a été corrompu mais qui reste intrinsèquement digne. Pic n'utilise pas le récit de la chute de l'homme, mais exploite plutôt la philosophie platonicienne car, en effet, ce sont l'ignorance et les vices qui ont obscurci la raison (Discours, p. 19). Dans l'Heptaple, qui est de 1489, alors que le Discours est de 1486, Pic traite aussi de la connaissance de soi (p. 138). L'approche générale reste la même, mais la connaissance de soi constitue maintenant le passage impératif vers toute autre connaissance. Se connaître, c'est rentrer en soi pour y découvrir Dieu. Pic reprend le passage du Cantique des cantiques où il est question de la connaissance de soi (I, $8:$ : Si tu t'ignores toi-même, $\hat{o}$ belle entre les femmes, sors et va-t-en après le train de tes troupeaux »). Pour lui, cela signifie que ne pas se connaître revient à suivre la bête qui est en soi (Heptaple, p. 194-5). Par la connaissance de soi, l'esprit ainsi purifié, l'homme rejoint la divinité dans l'époptéia, c'est-à-dire le rapport d'union intellectuelle de l'homme à Dieu (Discours, p. 29). Dans le schéma anthropologique exploité par Pic, une telle union est possible en raison de la sumpathéia, l'harmonie de l'univers due au rapport mutuel des natures entre elles (Discours, p.59). La clef de voûte de cette harmonie est l'homme, plus précisément sa nature : «car la nature de l'homme en est le lien [de la Nature] et comme le composé »(Discours, p. 31). Bien que la pensée de Pic se place toujours sur un plan allégorique, son point de vue sur la nature reflète une inflexion qui se retrouvera confirmée et affirmée dans l'attitude de penseurs comme Francis Bacon et plus tard dans tout l'essor scientifique du XVII ${ }^{\mathrm{e}}$ siècle. En effet, Pic voit en la Nature la source de toute connaissance (Discours, p. 61). Calvin, lui aussi, voit dans la Nature le lieu où se révèle la gloire de Dieu. Dans le théâtre de 
Shakespeare, la Nature a perdu ce caractère mystique. Elle constitue plutôt une force inquiétante et incompréhensible. L'action dramatisée révèle l'absence d'harmonie générale dont l'homme serait le noyau central. La nature se révèle complexe et trompeuse. Une forêt peut se déplacer et un homme peut ne pas naître d'une femme. Quant à la nature de l'homme, elle ne s'imbrique pas dans un ensemble. Il n'y a pas de sumpatheia. L'homme est dépeint seul, assailli de toute part avec pour seul ressource son libre arbitre.

Le libre arbitre est le sujet sur lequel Pic et Érasme se rapprochent le plus l'un de l'autre. Pour Pic, le libre arbitre, don de Dieu, peut être utilisé à mauvais escient. Il préconise donc une sainte ambition, c'est-à-dire le désir d'abandonner la médiocrité pour l'excellence (Discours, p. 13). Pic s'écarte donc de l'enseignement de saint Paul dans l'Épître aux Romains (chap. 7), où l'homme désire le bien mais cède au mal car il est l'esclave du péché. Chez Pic, l'homme peut trouver en lui la volonté de s'élever vers le bien. La place de l'homme dans la création illustre ce qu'est le libre arbitre : le libre choix de se diriger dans n'importe quelle direction, vers les dieux ou vers les bêtes, liberté qui a été voulue par Dieu (Discours, p. 7). Cependant, le monde, chez Pic est hiérarchisé en strates superposées. Le milieu du monde est donc le degré qui se trouve à mi-chemin entre ciel et terre. Deux remarques s'imposent. Pic recentre donc toute la problématique du bien et du mal sur l'homme seul. Deuxièmement, Pic conserve la notion d'une nature humaine simple qui serait par choix soit toute animale, soit divine. Ce n'est plus le cas chez Montaigne et dans le théâtre de Shakespeare. Si le choix de leurs actions incombe bien, au moins en partie, aux hommes, en revanche aucun des deux auteurs ne place l'homme à mi-chemin entre ciel et terre. Cette vision allégorique est abandonnée au profit d'une vision poétique. L'homme est considéré de façon plus réaliste comme une des créatures d'un vaste ensemble que constitue le monde. L'opposition entre le ciel et la terre est intériorisée dans la nature même de l'homme sous la forme du conflit entre ses aspirations morales, le ciel, et ses besoins physiques, la terre. Cette opposition ne peut être réduite et c'est la nécessité d'en tirer du sens pour lui-même qui fait de la conception de l'homme, chez Montaigne et Shakespeare, une conception poétique. Cette dernière modifie le sens qui était alors donné au libre arbitre. 
$\mathrm{Au} \mathrm{XVI} \mathrm{X}^{\mathrm{e}}$ siècle, la grande œuvre sur le libre arbitre est l'essai qu'Érasme lui consacre en 1524. Dès le début, Érasme donne du libre arbitre une définition qui recouvre exactement ce qu'en disait Pic de La Mirandole lorsque ce dernier insistait sur la notion de volonté : «nous définirons le libre arbitre comme un pouvoir de la volonté humaine grâce auquel l'homme peut s'appliquer à tout ce qui le mène au salut éternel, ou au contraire s'en détourner ${ }^{96}$. Érasme reprend une nouvelle fois le récit de la chute de l'homme. Dans son appréciation des conséquences de cette chute, il attribue à la volonté le statut d'entité réelle dans le cadre, comme toujours, d'un discours allégorique. Ainsi, la volonté d'Adam fut-elle corrompue par son amour immodéré pour Ève. Ainsi, par extension, la raison fut, elle aussi, corrompue. Ce qui signifie que la raison humaine est obscurcie par le péché ; mais elle n'est pas éteinte. Sans la nommer, Érasme fait référence à la notion théologique de synergie: Dieu accompagne toujours l'effort humain (Libre arbitre, p. 213). Par la grâce de Dieu, la volonté de l'homme a recouvré sa liberté. Puisque la raison humaine n'est pas entièrement éteinte, il reste donc possible à l'homme de choisir, en toute connaissance de cause, d'œu vrer pour son salut. Il y a donc interaction entre l'homme et Dieu. Érasme rejette l'idée d'une détermination universelle. Cette dernière aurait pour conséquence d'absoudre l'homme de toute responsabilité pour ses péchés. C'est l'argument principal des défenseurs du libre arbitre. Dans son introduction à l'essai d'Érasme, Pierre Mesnard souligne que la querelle «marqua la fin de l'humanisme chrétien sous sa première forme, la rupture du luthéranisme avec le libéralisme évangélique du pré-seizième siècle » (p. 200). L'expression la plus forte, parce que posée et réfléchie, de cette rupture se trouve chez Calvin, grand humaniste lui-même mais champion des Lettres Saintes seulement, notamment dans l'Institution de la Religion Chrétienne.

La première édition de l'IRC fut publiée en latin en 1535 et en français en $1541^{97}$. Sa vie durant, Calvin reprit et augmenta l'Institution. L'édition finale comprend cinq livres dont un de renvois, préparé par Calvin lui-même, pour permettre la consultation facile de

\footnotetext{
96 Érasme, Essai sur le libre arbitre, in La Philosophie Chrétienne, éd. Pierre Mesnard, Paris, Vrin, 1970, p. 212. Toutes les citations renverront à cette édition.

97 Calvin, Institution de la Religion Chrétienne (1541), éd. Jacques Pannier, Paris, Les
} Belles Lettres, 2e éd., 1961, p. xv. Les références datées de 1541 renverront à cette édition. 
l'ensemble. Elle fut publiée en 1560 en français. L'objectif principal de Calvin est l'enseignement de ce qu'il appelle «la vraie piété ». Calvin mourut en 1564, année de la naissance de Shakespeare et de Galilée. Montaigne avait trente et un ans lors de la mort de Calvin. Dans l'IRC Calvin abandonne l'interprétation allégorique des Saintes Écritures au profit d'une approche rationnelle. Le mot institution, qui signifie enseignement au $\mathrm{XVI}^{\mathrm{e}}$ siècle, indique l'objectif de l'œuvre. Dans l'Epistre au Roy (1535), c'est-à-dire au roi François I ${ }^{\text {er }}$, roi de 1515 à 1547, Calvin mentionne cet objectif dès les premières lignes (IRC 1541,

p.7). Cependant, cette adresse au roi a surtout pour objectif de défendre ceux que l'on persécutait pour leur «nouvelleté », mot repris par Calvin lui-même. Placé en tête de l'œuvre, l'« Argument du présent livre» est une adresse au lecteur, faite par Calvin à la troisième personne, pour lui expliquer le but de son entreprise. Plus tard, Montaigne, lui aussi, s'adressera directement au lecteur dans un esprit tout à fait différent, voire opposé. Dans l'« Argument», Calvin introduit plusieurs notions importantes. Elles forment un ensemble cohérent et révèlent un esprit nouveau quant à la façon de considérer la condition de l'homme. Premièrement, l'Écriture sainte contient une doctrine qui constitue un but en soi. Elle est donc une voie que le croyant doit suivre. Point important, cette voie se matérialise par l'appel du Saint Esprit. La notion de progrès s'affirme ici par le biais d'un but à atteindre et d'un moyen pour ce faire qui sont tous deux clairement définis. Deuxièmement, les simples doivent être conduits par la main par ceux qui ont reçu de Dieu «plus ample lumière». Troisième point, ce que Dieu nous a enseigné constitue une somme. Rien ne peut y être ajouté. Quatrième point, l'Écriture Sainte représente la «philosophie chrétienne », expression qui se trouve déjà chez Érasme. Cinquièmement, l'œuvre qui est présentée au lecteur se veut être une clef et ouverture pour donner accès à tous à l'entendement de l'Écriture Sainte. Calvin décrit aussi son œuvre comme une somme. Sixièmement, Calvin déclare avoir renoncé à tout abus de paroles. La lecture de son œuvre prouvera sa bonne foi. La même attitude se trouvera chez Montaigne. Septième point, Calvin introduit la notion de plan général pour son œuvre. Celle-ci est un chemin sur lequel le lecteur ne doit cesser d'avancer. L'œuvre ellemême représente l'expérience du chrétien qui progresse vers Dieu. Calvin utilise ici, déjà, la notion de progrès comme mouvement 
inéluctable vers la lumière. Le huitième et dernier point présente lui aussi un aspect moderne puisque Calvin recommande la vérification constante de la justesse de ses propos à l'aide des Écritures Saintes. C'est l'attitude que recommandera Francis Bacon pour l'étude de la nature.

Avant la lettre, le plan de Calvin est d'essence scientifique. Cependant, sa méthode ne constitue pas une rupture par rapport à ce que nous avons vu chez Pic de La Mirandole, mais plutôt, là aussi, une inflexion. Chez Pic toute la connaissance ou sagesse se trouve dans la nature et c'est elle qu'il faut étudier méthodiquement pour en révéler les secrets, témoins de la grandeur de Dieu et de la dignité de l'homme. Chez Calvin, l'objet d'étude est l'enseignement divin qui se trouve dans les Écritures Saintes. Le point de départ est différent chez l'un et l'autre auteur, mais ils partagent la même attitude d'esprit et le but ultime est identique, c'est-à-dire fonder la dignité de l'homme dans sa relation au divin. Cependant, bien que Calvin accentue les aspects négatifs de l'anthropologie issue du Moyen Âge, son humanisme, au sens propre du terme, apparaît dès les premières lignes de l'IRC (1541) lorsqu'il fait référence à la connaissance de soi (IRC 1541, p. 39). Calvin applique à un contexte chrétien le procédé de l'aporie, utilisé par Socrate dans les dialogues de Platon. Se connaître c'est, comme chez Érasme, mais de façon plus radicale, reconnaître la misère dans laquelle l'on se trouve, et alors, désirer tout ce qui nous manque, c'est-à-dire Dieu. La connaissance de cette misère "enflamme » l'homme du désir de Dieu comme la reconnaissance de leur ignorance conduisait les interlocuteurs de Socrate à désirer la vérité et à se mettre en quête d'elle. Or, la Vérité, pour le chrétien, c'est Dieu. C'est sur cette nouvelle façon d'interpréter le précepte delphique que Calvin fonde son anthropologie. Le mal, chez Pic et Érasme, est une erreur que l'homme commet. Cela implique que la nature originelle de l'homme a subi une modification avec la chute mais qu'elle n'a pas disparu. Faire le bien reste possible. L'homme a le choix. Chez Calvin, il en va autrement. La corruption est naturelle à l'homme : "Car notre nature n'est seulement vuide et destituée de tous biens, mais elle est tellement fertile en toute espèce de mal, qu'elle ne peut être oisive. Ceux qui l'ont appelée concupiscence n'ont point usé d'un mot trop impertinent, moyennant qu'on ajoutât ce qui n'est concédé de plusieurs : c'est que toutes les parties de l'homme depuis l'entendement jusques à la volonté, depuis 
l'âme jusques à la chair, sont souillées et du tout remplies de cette concupiscence, ou bien, pour le faire plus court, que l'homme n'est autre chose, de soi-même que corruption » (IRC 1541, p. 91-2). Dan s ce texte, nous retrouvons le mot clef de concupiscence. Nous avons vu comment Montaigne l'oppose à la raison, mais au détriment de cette dernière. D'autre part, il montrera aussi que la nature humaine n'est pas uniforme mais qu'elle varie d'un individu à un autre.

Plus bas, Calvin souligne que la responsabilité de sa chute incombe entièrement à l'homme. Ce faisant, il distingue entre ce qui est naturel à l'homme, c'est-à-dire la corruption, et la nature de l'homme, état qui est une qualité et non une substance (IRC 1541, p. 92 et 93). Calvin a renoncé à l'interprétation allégorique des Écritures Saintes, mais ici il introduit une différence entre un état naturel de l'homme, état corrompu et haï de Dieu, et un état idéal de l'homme, état premier que Dieu aime au même titre que toute Son œuvre. Cette division entre ces deux états n'est pas sans rappeler le monde idéal de Platon. Chez Pic de la Mirandole et chez Érasme, l'homme, image de Dieu (Genèse I, 26), conservait de ce fait un lieu avec Lui en dépit de la chute. Or, pour Calvin, cette image a été effacée (IRC 1541, p. 86) L'homme est donc dans un état d'aliénation au sens étymologique du terme. Il est en exil, étranger à Dieu par la chute, étranger au monde par son ignorance et à lui-même par sa vanité. Montaigne et Shakespeare reprennent ces éléments; mais les questions qu'ils impliquent pour l'homme ne peuvent attendre. Calvin représente l'homme dans une sorte de stase, en attente du retour vers Dieu, car cette vie est une mort. Chez Montaigne et Shakespeare l'homme est représenté irrémédiablement pris dans la dynamique de ses actes. Il ne peut faire l'économie de chercher les réponses aux questions générées par le simple fait de se trouver au monde.

Calvin place, lui aussi, l'homme au sein du monde. Il le fait par le biais de l'image classique selon laquelle il faut juger l'arbre aux fruits qu'il porte. Cependant, il modifie cette image pour ne parler que de racines pourries qui ne peuvent donner que des rameaux du même genre. Par son péché, Adam est à l'origine de cette pourriture (IRC 1541, p.89). Cette image indique où se trouve l'homme dans l'anthropologie de Calvin. Chez Pic de la Mirandole, chez Érasme, la position de l'homme se situe à mi-chemin entre les esprits et les bêtes. Calvin le place au sein du monde animal, sur la Terre qu'il compare à 
une forêt. Cependant, il n'abandonne pas complètement le procédé de distinctions par divisions qui est si fréquemment utilisé par les humanistes. Ainsi, il divise ce qu'il appelle l'âme humaine en intelligence et volonté. L'intelligence n'est pas aveuglée au point de n'avoir conservé quelque pouvoir. Calvin voit dans le désir de la vérité qui se manifeste chez les hommes, la preuve de cela (IRC 1541, p. 114). Ce passage n'est pas sans rappeler le début de la Métaphysique d'Aristote : «Tous les hommes désirent naturellement savoir ${ }^{98}$, et ce qui suit. Chez Calvin, cependant, cette intelligence, ou raison, ou encore entendement, s'égare souvent, en raison de la curiosité. Calvin aboutit très vite à une autre distinction, à savoir l'intelligence des choses terriennes, et celle des choses célestes (IRC 1541, p. 115). Pour cette dernière, lorsqu'il s'y applique, l'homme est vite convaincu de son «imbecillité». Calvin définit avec précision ce que sont les choses célestes: "la règle et raison de vraie justice et les mystères du Royaume céleste». Cette intelligence comprend «la connaissance de Dieu et de sa volonté, et la règle de conformer sa vie à icelle». Quant aux «choses terriennes», il les identifie tout aussi clairement: « J'appelle choses terriennes, lesquelles ne touchent point jusqu'à Dieu et son Royaume, ni à la vraie justice et immortalité de la vie future, mais sont conjointes avec la vie présente et quasi encloses sous les limites d'icelle». Puis il indique ce qu'elles contiennent : «la doctrine politique, la manière de bien gouverner sa maison, les arts mécaniques, la Philosophie, et toutes les disciplines qu'on appelle libérales». Implicitement, cette distinction entre «royaume de Dieu» et «vie présente» renvoie à la conception géocentrique de la Création. Copernic ne publiera sa théorie sur l'héliocentrisme qu'à la veille de sa mort, en 1543 et Galilée ne naîtra qu'en 1564. Calvin établit donc deux mondes distincts et qui semblent s'exclure, celui des hommes et celui de Dieu. Ce dernier, l'intelligence, aussi appelée raison humaine, ne peut le comprendre, le saisir. Chez Montaigne et chez Shakespeare, en revanche, Terre et Ciel fusionnent. Les « choses terriennes touchent jusqu'à Dieu» parce les actes des hommes ont des conséquences qui les dépassent mais les concernent néanmoins. Le roi de comédie, dans Hamlet, exprime cela ainsi : «Our thoughts are ours, their ends none of our own » (III.ii.188). Le royaume de Dieu est une réalité terrienne. Cependant, Calvin conserve l'image traditionnelle du lien de l'homme à

98 Aristote, La Métaphysique, (A, 1, 980 a 21), trad. J. Tricot, Paris, Vrin, 1986, p. 2 
Dieu qui se matérialise sous la forme du sentiment naturel de la divinité (IRC 1541, p. 43). Deuxième point, avec la distinction entre choses «terriennes » et choses « célestes » Calvin établit, le premier il semble, une nette différence de contenu entre la philosophie, qui fait partie des «choses terriennes », et la connaissance de Dieu qui est, bien qu'il ne la nomme pas, la théologie et renvoie à un monde supraterrestre. Montaigne rejette une telle distinction et place philosophie et théologie sur le même plan, dos à dos. Chez lui, la différence entre choses «terriennes» et choses «célestes»s'estompe parce que ces deux éléments représentent les deux parties d'un même élément, c'està-dire la vie humaine. Au début de l'essai «De l'amitié», l'image utilisée par Montaigne témoigne de cette représentation du monde: «Considérant la conduite de la besogne d'un peintre que j'ai, il m'a pris envie de le suivre. Il choisit le plus bel endroit et milieu de chaque paroi, pour y loger un tableau élaboré de toute sa suffisance; et, le vide tout autour, il le remplit de crotesques, qui sont peintures fantasques, n'ayant grâce qu'en la variété et étrangeté » (I, 28, 183a).

Le concept ici suggéré, et sur lequel repose tout l'essai, est celui du sumbolon, c'est-à-dire cet objet brisé en deux dont une partie est présente et l'autre absente mais néanmoins essentielle pour fonder la réalité totale de l'objet. C'est la raison pour laquelle Montaigne aime la poésie. Le poème, et ce qu'il suggère, forment à eux deux la représentation symbolique du monde et l'expression du tout. Le théâtre de Shakespeare se fonde sur la même représentation symbolique du monde. Les actes humains mis en scène entraînent immanquablement des conséquences qui dépassent «les choses terriennes », pour reprendre la terminologie de Calvin. La métaphore du monde comme scène de théâtre, et donc comme représentation, comme signe, est prise au pied de la lettre chez Shakespeare et constitue une autre preuve de la conception symbolique du monde qu'il partage avec Montaigne. Choses «terriennes» et choses «célestes» sont conjointes, à l'image des deux parties du sumbolon.

Le projet de se connaître, aboutit pour Montaigne à l'expérience de l'altérité radicale en lui-même, et du même coup du mystère, du divin. Sur ce point, Montaigne se trouve dans une opposition à Calvin qui est beaucoup plus radicale que celle des humanistes de la première partie du XVI ${ }^{\mathrm{e}}$ siècle. En effet, se connaître, chez Calvin, est impossible si l'on n'a pas d'abord appris à connaître le Christ (IRC 1541, p. 40). 
Avec l'exemple de Socrate, Montaigne prend la position exactement inverse. C'est en s'étudiant que l'homme contient son animalité et s'élève vers la divinité. Quelques lignes plus haut, Calvin écrit qu'il faut se déplaire à soi pour aspirer au Seigneur (IRC 1541, p. 40). Sur ce point, encore, Montaigne se situe aux antipodes de Calvin. Il faut apprendre à s'aimer et à aimer la vie. Montaigne se place dans la tradition de la philautia antique. S'aimer, c'est aimer l'image de Dieu et, ainsi, aimer Dieu. Enfin, la volonté et le libre arbitre représentent deux autres éléments importants chez les humanistes pour fonder la dignité de l'homme. Calvin reprend cette argumentation et lie volonté et libre arbitre: "Or quand on assigne libéral arbitre à l'homme, combien y en a-t-il qui ne conçoivent incontinent qu'il est maître, et de son jugement, et de sa volonté, pour se tourner de sa propre vertu et d'une part et d'autre?»(IRC1541, p. 108). Ces lignes renvoient clairement à l'argumentation des humanistes. Après une longue démonstration, Calvin lie volonté, liberté et libre arbitre pour leur nier toute réalité positive en dehors du cadre naturel. Le désir naturel de faire le bien que les philosophes prêtent à l'homme vise la satisfaction des sens, ce qui est commun aux bêtes. L'homme suit alors sa nature terrestre et non celle qu'il a reçue de Dieu. Calvin distingue donc entre deux types de raison humaine. L'une est «naturelle», commune aux bêtes. Elle est à l'origine de sa «volonté», ce mouvement pour la satisfaction des sens. Elle ne peut en rien fonder le libre arbitre puisqu'elle est l'esclave des besoins corporels de l'homme. Le second type de raison est immortel et c'est la seule «droite raison » (IRC 1541, p. 130-1). La volonté ne peut être que mauvaise parce qu'elle est « en servitude de péché»(IRC 1541, p. 140). Certes, l'homme manifeste toujours de la volonté, mais comme lui elle a subi la corruption de la chute $(I R C, 1541$, p.140). Calvin insiste sur le fait que le péché chez l'homme n'est pas le résultat de l'ignorance, comme Érasme le soutenait à la suite de Platon. Au contraire, en raison de sa nature corrompue, l'homme pèche volontairement, par nature. L'aparté d'Hamlet «I must be cruel only to be kind», tel que nous le comprenons, se rattache à la position exprimée ici : «Il nous faut donc observer cette distinction : c'est que l'homme après avoir été corrompu par sa chute, pèche volontairement, et non pas malgré son cœur, ni par contrainte ; qu'il pèche, par une affection très encline, et non pas étant contraint de violence ; qu'il pèche du mouvement de sa propre cupidité, 
et non pas étant contraint d'ailleurs; et néanmoins que sa nature est si perverse, qu'il ne peut être ému, poussé, ou mené, sinon au mal» (IRC 1541, p. 141). Montaigne et Shakespeare donneront une image bien plus complexe de l'homme. Enfin, dans le droit fil de la pensée de saint Paul exprimée dans l'Épître aux Romains (chap.7), Calvin conclut: "Je pense que nous avons suffisamment prouvé, comme l'homme est tellement tenu captif sous le joug de péché, qu'il ne peut, de sa propre nature, ni désirer le bien en sa volonté, ni s'y appliquer » (IRC 1541, p. 158). Cependant, cela ne signifie pas qu'il rejette toute notion de dignité humaine. Cette dernière est une réalité, mais elle se fonde en Dieu, et non en l'homme.

En 1965, Eleanor Prosser publia un article dans Shakespeare Studies intitulé «Shakespeare, Montaigne, and the Rarer Action » ${ }^{99}$, où elle mettait en évidence plusieurs parallèles entre le début de l'essai « De la cruauté» dans la traduction de Florio et les vers prononcés par Prospéro au dernier acte de La Tempête (v.i.25-28). Cependant, elle concluait que Shakespeare ne partageait pas le point de vue de Montaigne sur la vertu. Il nous semble que Eleanor Prosser a tout à la fois raison et tort. Elle a raison sur la pertinence des parallèles ; mais il n'en est pas de même pour le deuxième point. En effet, Montaigne soutient, avec l'exemple de Socrate, que la vertu la plus parfaite s'accomplit en dehors de tout effort car, si cela n'était pas le cas, alors la vertu serait dépendante du vice. La vertu est affaire de connaissance de soi. C'est par la connaissance de lui-même que Socrate se dégage de ses vices naturels. Dans La Tempête, Shakespeare partage le même point de vue que Montaigne. Il a tiré du début de l'essai certaines tournures mais il suit l'esprit des arguments de Montaigne tels qu'ils apparaissent plus loin à propos de Socrate. De façon schématique, Érasme oppose la vertu au péché, Pic la dignité à la corruption, Calvin, la grâce à la corruption. Dans l'essai «De la cruauté», Montaigne oppose cruauté et vertu socratique. La notion de cruauté a remplacé l'état de péché que les autres penseurs attribuent à l'homme.

99 Eleanor Prosser, «Shakespeare, Montaigne, and the Rarer Action », in Shakespeare Studies, vol. I, 1965, p. 261. 
Montaigne et Shakespeare se situent à mi-chemin entre les thèses des humanistes et celles de Calvin. Des éléments des deux bords se retrouvent chez eux mais ils subissent une inflexion qui représente un dépassement des querelles de la première moitié $d u X V I^{e}$ siècle. La relation à Dieu n'est plus conçue de Dieu vers l'homme mais de l'homme vers Dieu. Elle n'est plus extériorisée mais intériorisée. La poésie remplace l'allégorie. Elle crée un sens qui dépasse la simple signification des mots et se donne comme image du monde. En effet, l'association du signifiant au signifié renvoie à l'image du sumbolon. L'homme est la partie présente du sumbolon, la partie absente est le plan sur lequel il fonde sa dignité. Cette seconde partie représente un acte de foi, l'expression de la volonté de l'homme. L'ensemble constitue son humanité. En cela, il me semble, Montaigne et Shakespeare reprennent l'image néo-platonicienne que l'on retrouve aussi dans l'Heptaple de Pic de La Mirandole et selon laquelle le ciel est cercle et l'âme humaine aussi. L'image du monde représenté par le cercle se trouve, par exemple, dès l'acte I, scène deux d'Hamlet (I.ii.125-128). L'homme ne se trouve pas entre les esprits purs et les animaux; mais plutôt au sein du monde réel. L'homme n'est pas composé d'une nature double, c'est-à-dire d'un corps corrompu et d'une âme divine. Montaigne et Shakespeare abandonnent le procédé de divisions successives que l'on trouve chez Pic ou Érasme ou encore Calvin mais à un moindre degré. L'homme est une « complexion», c'est-à-dire un ensemble d'éléments disparates qu'il a reçu de la nature sans qu'il sache ni pourquoi ni comment. Il a donc l'obligation d'apprendre à se connaître car il se méconnaît. Il n’y a pas de séparation entre les «choses terriennes» et les «choses célestes», pour reprendre la terminologie de Calvin. Chez Montaigne et dans le théâtre de Shakespeare elles sont conjointes. Les actes des hommes ont toujours des conséquences qui les dépassent ou, pour le dire autrement, la réalité même de son existence échappe à l'homme. Le précepte delphique qui exhorte à se connaître soi-même n'a pas pour objet de fuir ses propres vices mais plutôt de les connaître afin d'apprendre à les ménager. C'est dans la mise en œuvre de ce précepte que l'on recouvre sa divinité par une haute et divine « résolution » chez Montaigne et par «a rarer action» chez Shakespeare. Il ne s'agit pas d'une transformation de la nature humaine mais, à la suite de Pic et de la tradition dont il s'est lui-même inspiré, il s'agit d'une transformation 
spirituelle à l'image de l'epopteia, le rapport d'union intellectuelle de l'homme à Dieu. Dignité et divinité de l'homme se confondent. Cependant, cela conduit à une conséquence importante. Le concept de dignité générale de l'homme, cher à Pic de La Mirandole, ne se trouve pas chez Montaigne et Shakespeare. La dignité est le produit de la volonté individuelle.

Ainsi, et pour répondre à la question du colloque, Montaigne et Shakespeare représentent effectivement un nouvel humanisme par rapport à celui de la première moitié $\mathrm{du} \mathrm{XVI}^{\mathrm{e}}$ siècle. Cet humanisme repose sur la ré-appréciation du précepte delphique et aboutit à la représentation de l'existence comme sumbolon. L'homme a un devoir d'humanité. L'humanisme de Montaigne et Shakespeare constitue une poétique de la vie. La dignité de l'homme se joue sur la partie abstraite, absente, immatérielle du sumbolon. Dans son théâtre, Shakespeare manifeste la conscience tout à la fois de l'extrême fragilité de ce second plan et de son caractère indispensable pour l'homme.

Ce nouvel humanisme, pour lequel la notion de devoir est centrale en raison de l'obligation du connais-toi toi-même, n'a pas survécu à la révolution scientifique qui débute au XVII ${ }^{\mathrm{e}}$ siècle, ni au Siècle des Lumières. L'humanisme moderne se trouve aux antipodes de celui de Montaigne et Shakespeare parce qu'il s'appuie sur le droit écrit, c'est-à-dire la croyance qu'il est possible de matérialiser sous forme de lois le plan intangible sur lequel l'homme fonde sa dignité.

Jean-François CHAPPUIT Université de Versailles-Saint-Quentin-en-Yvelines. 